\title{
Special issue on the 8th International Conference on Molecular Imprinting: MIP2014
}

\author{
Peter A. Lieberzeit
}

Received: 4 January 2015/Accepted: 5 January 2015/Published online: 6 February 2015

(C) Springer-Verlag Wien 2015

For roughly the last 30 years, chemistry has gained increasing understanding of the importance of interactions between individual molecules in addition to those leading to new chemical compounds. Artificial recognition systems both for analytical and catalytic purposes have evolved from this understanding and continue attracting increasing scientific interest. When it comes to straightforward synthesis of such systems, molecular imprinting into polymers seems unparalleled, because self-organization between monomers and a template molecule determine the steric and functional features of the final recognition cavity in the material. Despite great efforts and success, the field is still subject of substantial research, both for better understanding the molecular mechanisms of the process and for finding innovative ways to push molecularly imprinted polymers (MIP) forward towards production/synthesis on an industrial scale.

The conference series "International Conference on Molecular Imprinting" biannually brings the MIP community together and thus constitutes the largest scientific meeting in this specific area of Materials Science. In 2014, it was been held for the 8th time and organized by the group of Prof. Songjun Li at Jiangsu University in Zhenjiang 18-21 September. It saw a large number of participants including the pioneers of MIP, Prof. Guenter
Wulff and Prof. Klaus Mosbach, and many of the top groups and group leaders in today's MIP community. Organizing this meeting in China was a very logical choice, because MIP offer low-cost approaches on the way to high selectivity and are hence especially attractive for rapidly developing economies, such as the Chinese.

This special issue of "Monatshefte der Chemie" collects some of the papers presented at the conference. Others can be found in the Journal of Molecular Recognition, Key Engineering Materials, Journal of the Chinese Advanced Materials Society and Molecular Imprinting. Knowing that the topic is not fully in line with the priorities of this Journal-which of course usually focuses more on (organic) synthesis, let me nonetheless invite you, dear readers, into this highly interesting area on the border between Polymer Chemistry, Materials Science, Catalysis and Analytical Sciences. Experience, how seemingly straightforward synthesis leads to surprising materials properties and a multitude of matrices ranging from bulk polymers to nanoparticles including thin films. With this, I thank the organizers of the conference for a perfect event, the authors for their submission and the editors of the "Monatshefte" for their constant support and help in bringing together this special issue.
P. A. Lieberzeit $(\square)$

Department of Analytical Chemistry, Faculty for Chemistry, University of Vienna, Waehringer Strasse 38, 1090 Vienna, Austria

e-mail: Peter.Lieberzeit@univie.ac.at 